\title{
Análisis de la configuración del campo de estudio de la desigualdad educativa
}

\section{Configuration Analysis of the field of study in educational inequality}

\section{Diana Lorena Pineda-Ospina \\ Universidad Del Valle, Cali, Colombia}

\section{Resumen}

Objetivo: Analizar la configuración de la estructura intelectual del estudio de la desigualdad educativa. Metodología: Se diseñó una estrategia metodológica mixta a partir del diseño de un ejercicio bibliométrico. Desde la perspectiva cuantitativa, se desarrolló el procesamiento estadístico de la producción de documentos científicos de las bases de datos Scopus e ISI Web of Science en el campo de la desigualdad educativa y se construyeron y analizaron indicadores bibliométricos que permitieron la evaluación del rendimiento científico, su desarrollo y evolución. Desde la perspectiva cualitativa, se realizó el análisis documental, semántico y de contenido de un conjunto de documentos identificados. El procesamiento de la información se realizó en los softwares Modeler SPSS, Atlas ti, SciMAT y Knime. Resultados: Se identifica el campo de la desigualad educativa como consolidado, donde se destaca un mayor desarrollo analítico y metodológico desde finales de los noventa donde se incorporan distintas disciplinas al estudio que han enriquecido las perspectivas analíticas. Conclusiones y discusiones: La estructura intelectual del campo de la desigualdad educativa se caracteriza por su alto rendimiento y visibilidad, donde disciplinas como la economía, la psicología o las humanidades han aportado significativamente al desarrollo metodológico, teórico y práctico, generando un abordaje transdisciplinar que contribuye significativamente al estudio de la desigualdad social desde enfoques como el análisis multinivel, el curso de vida o el desempeño educativo.

Palabras clave: Bibliometría, Ciencias sociales, desigualdad educativa, desigualdad social.

\begin{abstract}
Objective: To analyze the configuration of the intellectual structure of the study in educational inequality. Methodology: A mixed methodological strategy was designed, based on the design of a bibliometric exercise. From the quantitative perspective, the statistical process of the production of scientific documents, which was based on the Scopus and ISI Web of Science databases in the field of educational inequality was developed. Likewise, bibliometric indicators were built and analyzed, which allowed the evaluation of scientific performance, its development and evolution. From the qualitative perspective, the documentary, semantic and content analysis of a set of identified documents was performed. The information processing was carried out in the Modeler SPSS, Atlas ti, SciMAT and Knime software's. Results: The field of educational inequality is identified as consolidated with greater analytical and methodological development in the late nineties. Since then, different disciplines are incorporated into the study, which have enriched the analytical perspectives. Conclusions and discussions: The intellectual structure in the field of educational inequality is characterized by its high performance and visibility, where disciplines such as psychology or the humanities have made a significant contribution to methodological, theoretical, and practical development, generating a transdisciplinary proposal. This contributes significantly to the study of social inequality based on approaches such as multilevel analysis, life course or educational performance.
\end{abstract}

Open Access:

ISSN: $0124-2121$ E-ISSN: $2665-2420$

\author{
ARTÍCULO DE INVESTIGACIÓN \\ CIENTÍFICA \\ Copyright $(\subseteq)$ \\ By Educación y Humanismo \\ Editor: \\ Dhayana Fernández Matos
}

Correspondencia: diana.pineda@correounivalle. edu.co

Recibido: 19-11-2020 Aceptado: 15-03-2021 En línea desde: 08-07-2021

Keywords: Bibliometry, educational inequality, social inequality, Social sciences. 


\section{Introducción}

La literatura sobre el estudio de la desigualdad educativa en las ciencias sociales se ha orientado, básicamente, en dos perspectivas analíticas. La primera de ellas, desde el enfoque de la reproducción social y cultural, hace énfasis en el rol de la escuela para la reproducción de las desigualdades y los efectos de las asimetrías como resultado del capital cultural acumulado por los estudiantes. En este enfoque, se señala el papel de la educación para mantener y perpetuar las relaciones asimétricas en lo económico, social y cultural, donde la educación, como instancia social subordinada, responde a las necesidades de mantenimiento de la desigualdad.

Para Bourdieu \& Passeron (2009), la escuela se constituye como el espacio de reproducción de las desigualdades en el que se pone en juego las distintas acumulaciones de capitales y cumple una función ideológica y legitimadora de la reproducción de las desigualdades sociales. En su desarrollo, los autores analizan cómo la acción de los factores culturales opera ante la educación y cómo el origen social es la mayor fuerza que influye sobre el medio estudiantil, llevando a la eliminación, el relegamiento y/o el retraso en el ciclo académico. En efecto, para ellos, la paradoja señala que los más desfavorecidos culturalmente sufren más su desventaja allí mismo donde son relegados como consecuencia de sus desventajas (Bourdieu \& Passeron, 2009).

En contraste, el enfoque de la acción social surge como una forma alternativa a las explicaciones culturalistas que permita identificar otros efectos distintos al de la clase social por la vía familiar. En este enfoque se relaciona la desigualdad de los resultados educativos con los diferencias de recursos materiales y de habilidades cognitivas. Para ello, propone romper con las carácter funcionalista y teleológico para identificar los micro fundamentos sólidos en el análisis de la acción individual y de las consecuencias intencionales e imprevistas en los que se ve a los individuos como miembros de clase, sujetos a los diferentes niveles y formas de oportunidades y constricciones que implican sus distintas situaciones de clase (Boudon 1983; Goldthorpe, 2010).

Estos dos enfoques teóricos describen las orientaciones de mayor tradición en la literatura de las desigualdades educativas en las ciencias sociales. Sin embargo, en esta perspectiva surgen los interrogantes sobre ¿cuáles son los rasgos y características que han configurado el campo de estudio de las desigualdades educativas en las últimas décadas?, ¿cuáles son los autores, instituciones, revistas, y descriptores que se destacan el en campo a lo largo del tiempo? y ¿cómo se ha configurado la evolución del campo de estudio?

Para responder a estas inquietudes, el artículo tiene como propósito analizar la estructura intelectual de la desigualdad educativa en las últimas décadas. Metodológicamente, se diseñó una estrategia mixta. Desde el enfoque cuantitativo, se procesó una muestra de 
documentos científicos publicados en las bases de datos Scopus e ISI Web of Science que permitió el desarrollo de un ejercicio bibliométrico y la construcción y análisis de indicadores para medir la evaluación del rendimiento científico, su desarrollo y evolución. Desde la perspectiva cualitativa, se desarrolló análisis documental, semántico y de contenido sobre los documentos identificados. El procesamiento de la información se realizó en los programas Modeler SPSS, Atlas ti, SciMAT y Knime, los cuales permiten caracterizar la evolución del campo en las últimas cinco décadas.

\section{Marco teórico}

En la revisión de la literatura en ciencias sociales de las últimas décadas sobresale una vertiente orientada a integrar el marco analítico de dos perspectivas analíticas: la teoría de la reproducción cultural y la acción social. Ambos enfoques como marco interpretativo de la desigualdad educativa en el contexto actual de las sociedades industrializadas. Cada una de estas perspectivas analíticas plantea diferentes factores para comprender la incidencia de la desigualdad educativa, otorgando distintos niveles de significancia, constituyendo así un marco analítico para su interpretación.

Por una parte, la función determinista del origen social asigna un lugar futuro al estudiante, definiendo su destino académico y eliminando su capacidad de elección o reconocimiento de otras opciones. Por otra parte, el análisis costo beneficio adjudica a la decisión educativa una función utilitarista para evitar el descenso social, dejando de lado el entorno cultural y la historia de quien toma la decisión. Considerando que estos enfoques han sido opuestos dado su objeto de estudio y la limitación analítica de cada uno, autores como Breen y Jonsson (2005), Van de Werfhorst y Mijs (2007), y Boone y Van Houtte (2013), entre otros, proponen abordar la teoría de la reproducción cultural y la acción social como complementarias para la comprensión de la desigualdad educativa.

Breen y Jonsson (2005) analizan la importancia de las circunstancias socioeconómicas de los padres y cómo las características de la familia de origen están asociadas con los resultados educativos y del mercado laboral. Para los autores, sus hallazgos sobre las características socioeconómicas contribuyen al planteamiento de modelos de análisis de micro nivel donde se destacan los de elección racional. Con este abordaje analítico, resalta un mayor interés en ir más allá de la descripción en investigación de estratificación, realzando el rol explicativo a nivel individual la reproducción cultural.

Al analizar reproducción cultural y aversión al riesgo relativo como mecanismos explicativos para la desigualdad educativa, Van de Werfhorst y Mijs (2007) analizan cómo la integración de las variaciones en el resultado académico (producto de las diferencias culturales entre clases sociales y la aversión al riesgo relativo para evita la movilidad descendente) facilita la interpretación integral de los factores que intervienen en la desigualdad educativa, ampliando la capacidad de comprensión de la misma según el lugar en el que se produce. 
Boone y Van Houtte (2013) abordan la teoría de la reproducción cultural, el capital social y la teoría de la acción racional al investigar los mecanismos que conducen a las diferencias de clase social en elección educativa, identificando la forma cómo los alumnos perciben su proceso de elección académico en el marco de su concepción del sistema educativo y las opiniones de los padres sobre qué alternativas educativas son aceptables y cuáles no.

Esta exploración de estudios que integran los enfoques de la reproducción cultural y la teoría de la acción social dan cuenta de la capacidad explicativa de abordar en el análisis estas perspectivas para tener una mayor compresión de la desigualdad educativa en contextos específicos. Sin embargo, los estudios no permiten identificar las características del campo de estudio de la desigualdad educativa. Para ello, el ejercicio que se presenta en las siguientes secciones busca contribuir a la comprensión de la estructura intelectual de la desigualdad educativa.

La estructura intelectual de un campo de estudio es la identificación y análisis de las principales características, las áreas del conocimiento que han aportado a su desarrollo, sus principales enfoques y posibles derivaciones en el tiempo a partir de técnicas bibliométricas como el análisis de co-citaciones, mapas estratégicos o redes temáticas. En este sentido, la configuración de la estructura intelectual permite identificar cómo se ha desarrollado un campo de estudio en el tiempo, con enfoque retrospectivo, y cuáles son sus posibles desarrollos en el tiempo, con enfoque prospectivo (White \& Griffith, 1981; Ramos-Rodríguez \& Ruíz-Navarro, 2004; Nerur, Rasheed \& Natarajan, 2008; Pilkington \& Meredith, 2009).

De acuerdo con el objetivo del análisis propuesto, identificar la estructura intelectual de la desigualdad educativa contribuirá a la comprensión del campo de estudio en un sentido amplio, donde se recogen los rasgos de su desarrollo a partir de la depuración de documentos científicos con técnicas bibliométricas.

\section{Método}

El desarrollo metodológico para el objetivo propuesto parte de la realización de un ejercicio de análisis bibliométrico que permitió el análisis y mapeo científico de la estructura intelectual del campo de la desigualdad educativa. Con este fin, el análisis bibliométrico se orientó a la identificación de los principales indicadores de rendimiento que estructuran el campo desde la década de los setenta (Cobo, López-Herrera, Herrera-Viedma \& Herrera, 2011; Martínez, Cobo, Herrera \& Herrera-Viedma, 2015; Cobo, Martínez, Gutiérrez-Salcedo, Fujita \& Herrera-Viedma, 2015).

La estrategia metodológica diseñada se caracterizó por ser mixta. Desde la perspectiva cuantitativa, se incorporó el procesamiento estadístico de la producción de documentos científicos publicados en las bases de datos Scopus e ISI Web of Science en el campo de la desigualdad educativa. La identificación, procesamiento y depuración de la muestra de 
documentos científicos se realizó a través de ecuaciones de búsqueda estructurada cuyo centro analítico fue desigual educativa.

Se eligieron las bases de datos Scopus e ISI Web of Science como principal fuente de información debido a su reconociendo en el ámbito académico por la rigurosidad y calidad intelectual de sus publicaciones. En total, el tamaño de la muestra fue de 1.582 documentos. Cada uno de ellos se procesó con todas las variables asociadas a la depuración de la muestra de documentos científicos según metadatos como: información de la cita, información bibliográfica, resumen y palabras claves y referencias. Debido al tamaño de la muestra, este procesamiento se desarrolló en el software Modeler SPSS de IBM. La incorporación de Modeler SPSS como software para realizar la minería de datos fue posible gracias a la capacidad de procesamiento del programa que brinda ventajas del uso de algoritmos estadísticos sin programación. Como resultado del análisis cuantitativo, se construyeron indicadores bibliométricos, cadenas de clústers, mapas temáticos y mapas estratégicos que configuran la estructura intelectual de la desigualdad educativa a través del análisis de copalabras, autores, instituciones y jornales.

Posterior al procesamiento de la información estadística, se construyeron y analizaron indicadores bibliométricos que permitieron la evaluación del rendimiento científico, su desarrollo y evolución. El desarrollo de este análisis se realizó en el software Modeler SPSS y SCIMAT. El software SCIMAT se caracteriza por ser un programa para analizar la evolución del conocimiento científico. Como complemento al desarrollo cuantitativo, desde la perspectiva cualitativa, se incorporaron técnicas de análisis documental, semántico y de contenido sobre los documentos identificados, con los softwares Atlas ti y Knime, permitiendo identificar las principales características de la evolución temática del campo. La incorporación de los softwares Atlas ti y Knime facilitó la depuración de metadatos para la identificación de las sendas de desarrollo del conocimiento en el campo de la desigualdad educativa.

En conjunto, el desarrollo del análisis cuantitativo y cualitativo contribuyo a identificar la configuración de la estructura intelectual de la desigualdad educativa (López-Herrera, Cobo, Herrera-Viedma, Herrera, Bailón-Moreno y Jiménez-Contreras, 2009; Cobo, López-Herrera, Herrera-Viedma y Herrera, 2012). Como resultado del análisis, en la siguiente sección se presentan los aspectos centrales de la estructura intelectual del campo de la desigualdad social. Posteriormente, se presentan las principales conclusiones y discusiones del análisis donde se plantean las perspectivas analíticas en el campo de la desigualdad educativa en las ciencias sociales.

\section{Resultados}

El análisis de la estructura intelectual del campo de la desigualdad educativa se presenta en cuatro sección: i. Se analizan los documentos según el tipo, fuente, países y convergencia 
en áreas del conocimiento; ii. Se analiza la granularidad de los autores, donde se identifica su impacto y visibilidad a lo largo del tiempo; iii. Se analizan las palabras claves, o descriptores, del campo; y, iv. Se identifican los ejes temáticos centrales del estudio de la desigualdad educativa.

\section{Análisis por tipo de documento, fuente, países y convergencia en áreas del conocimiento}

El análisis de las desigualdades educativas como campo de estudio en las publicaciones científicas inicia en 1974 y sigue una tendencia creciente hasta el presente. A 2019, se publicaron mil quinientos ochenta y dos (1.582) documentos, de los cuales $82.5 \%$ son artículos, 9.2\% son libros o capítulos de libros y el $8.3 \%$ restante son conferencias, editoriales, revisiones o notas. Para la ventana de observación establecida, se identifica un crecimiento a lo largo del tiempo en la producción de documentos científicos, esta tendencia se da paralelo a ciclos en el nivel de impacto de la producción científica. Entre 1974 y 1997 se observan ciclos expansivos en la acumulación de citas, seguidos del período 2002 - 2010 y una caída en las citas a partir de 2011. A lo largo de los cuarenta y cinco años de estudio, se obtiene un promedio de citas de 36 y 474 documentos por año (gráfico 1).

Gráfico 1.

Documentos publicados entre 1974 y 2019

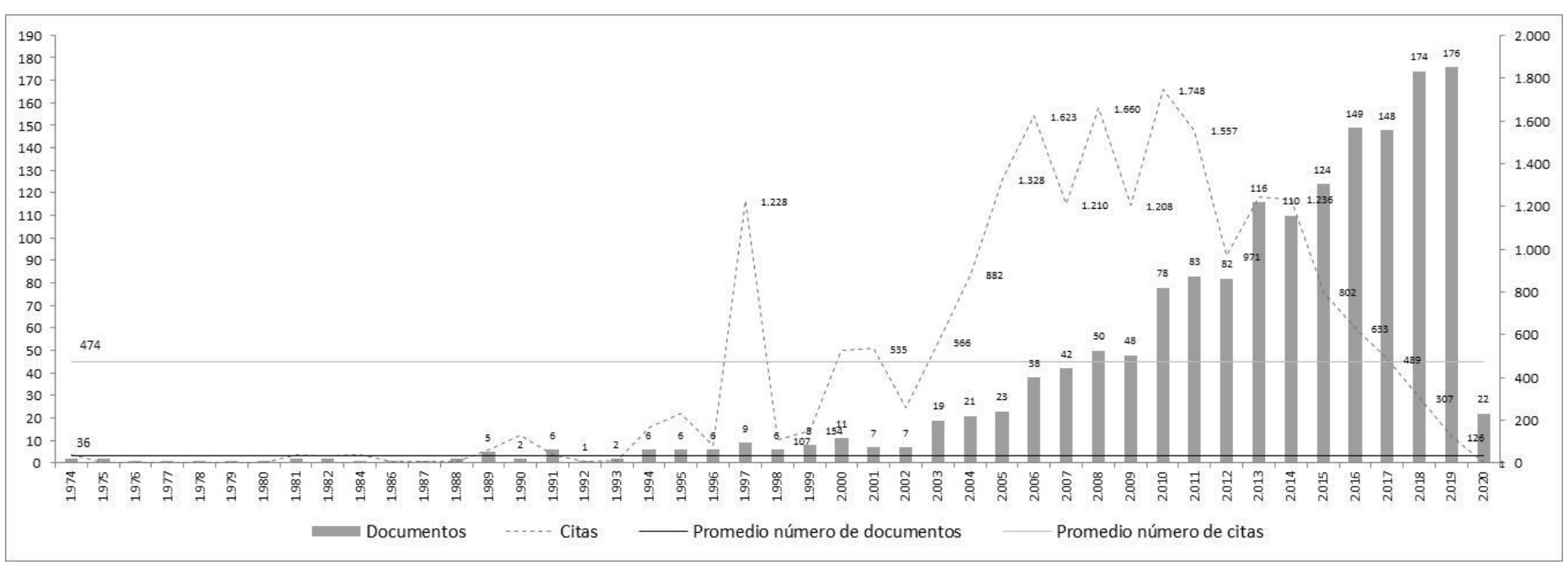

Nota. Elaboración propia (2021)

El primer documento publicado en el campo fue el artículo The causes of educational inequality: Further observations escrito por Raewyn Connell, de la Universidad de Sydney, Australia, publicado en el Journal of Sociology en 1974. Este artículo se caracterizó por ser un estudio comparativo sobre perspectivas analíticas de las desigualdades educativas en estudios secundarios en el campo.

La década de los setenta se caracterizó por su bajo nivel de publicaciones, acumulando 
ocho (8) documentos y cuarenta y cuatro (44) citas, en ocho (8) revistas diferentes. En esta primera década de publicaciones de documentos primarios, o centrales en el campo de la desigualdad educativa, el documento con mayor citación fue el artículo The politics of school busing del profesor Jonathan Kelley de la Universidad de Yale (Estados Unidos) en 1974 y publicado en Public Opinion Quarterly, donde recibió veintitrés citas (23).

Las décadas de los ochenta y noventa se caracterizaron por un bajo nivel de publicaciones de documentos en el campo. Se publicaron sesenta y siete (67) documentos. Sin embargo, en este período se acumularon dos mil doscientas sesenta y cuatro (2.264) citas. Los artículos más citados fueron: Population increase, economic growth, educational inequality, and income distribution. Some recent evidence, publicado por Rati Ram de la Universidad de Illinois (Estados Unidos) en el Journal of Development Economics en 1984 que recibió cuarenta (40) citas y, Explaining educational differentials: Towards a formal rational action theory, publicado en Rationality and Society, en 1997, por Richard Breen (Universidad de Oxford, Reino Unido) y Jhon H. Goldthorpe (Universidad de Oxford, Reino Unido), que acumuló 850 citas.

Entre 2000 y 2009 se publicaron doscientos sesenta y seis (266) documentos con nueve mil setecientos noventa y seis (9.796) citas. El artículo Inequality of opportunity in comparative perspective: Recent research on educational attainment and social mobility fue el más citado (556), publicado en el Annual Review of Sociology por Richard Breen (Universidad de Oxford, Reino Unido) y Jan Jonsson de la Universidad de Estocolmo en 2005.

Finalmente, entre 2010 y 2019, se configuró el período de mayor producción de documentos científicos, se alcanzó un total de mil doscientos cuarenta y uno (1.241) publicaciones y nueve mil ciento once (9.111) citas. El número de publicaciones de esta década es cuatro punto seis (4.6) veces las publicaciones del período pasado y ciento cincuenta y cinco (155) veces más que la primera década en la que surge el campo de estudio.

Para este período, el documento más citado fue el libro Whither opportunity: Rising inequality, schools, and children's life chances con trescientas quince (315) referencias, escrito por Greg J. Duncan (Universidad de California, Estados Unidos) y Richard J. Murnane (Universidad de Harvard, Estados Unidos) y publicado por la editorial Russell Sage Foundation en 2011. Al comparar el impacto del número de publicaciones según el número acumulado de citas, la década de los noventa fue el período de mayor disrupción en el campo; en promedio por cada documento publicado se alcanzó 16.3 citas, seguido de la década de los ochenta con 4.5 citas por documento, la década de los setena con 2.9 citas por documento, la década del 2000 al 2009 con 2.1 citas por documento y la década del 2010 al 2019 con 0.3 citas por documento. Estos datos indican que, a nivel de décadas, la mayor producción de documentos científicos ha generado un menor impacto en la comunidad científica. 
Respecto a las fuentes de los documentos, se identificaron seiscientos sesenta y cinco (665) revistas indexadas como fuente de artículos publicados. Del total, las diez revistas que han publicado el mayor número de artículos, que acumulan el $35 \%$ del total durante el período son: el British Journal of Sociology of Education, con el mayor número de artículos (40) en los 45 años de estudio, seguido de Research in Social Stratification and Mobility (32), Kolner Zeitschrift fur Soziologie und Sozialpsychologie (28), European Sociological Review (23) y International Journal of Educational Development (22), Zeitschrift fur Soziologie (21), Education Policy Analysis Archives (18), Educacao e Sociedade (17), Social Science Research (17) y Sociology of Education (16) (gráfico 2).

\section{Gráfico 2.}

Journals por acumulado de publicaciones por décadas

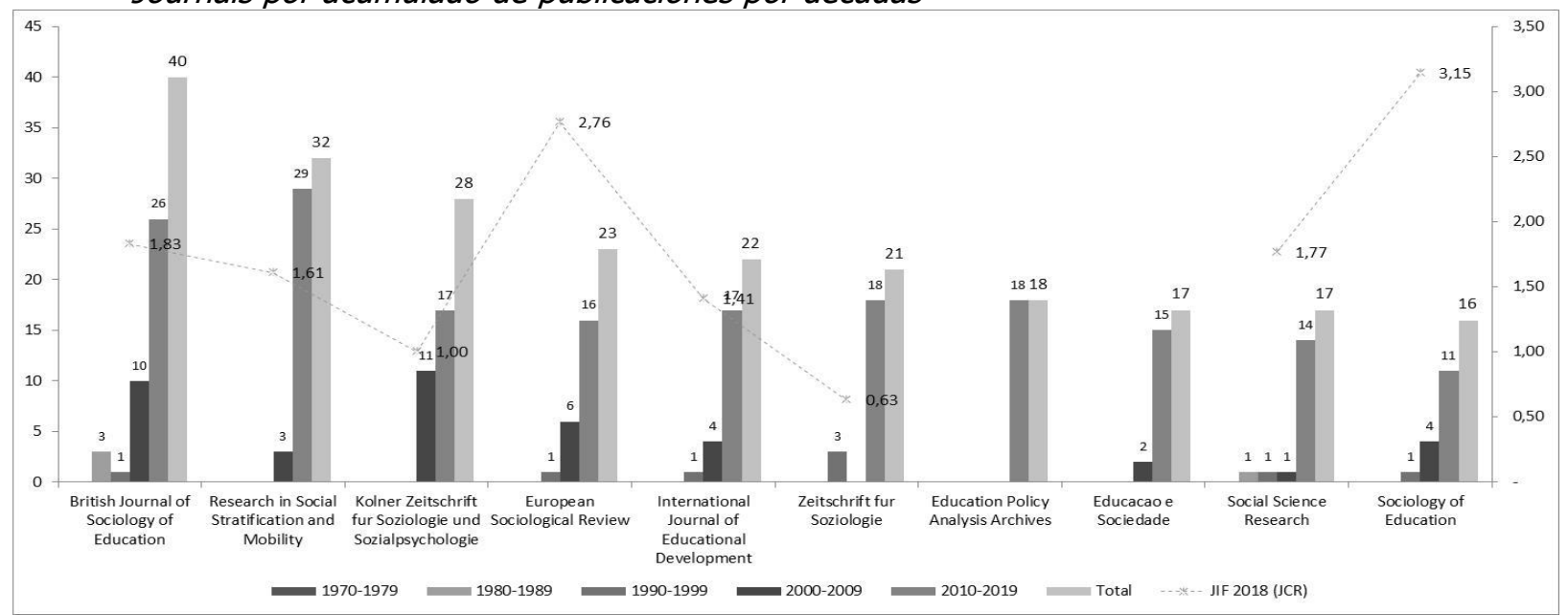

Nota. Elaboración propia (2021)

De acuerdo con el Journal Impact Factor - 2018 (JCR), la revista con mayor impacto en el campo del conocimiento es Sociology of Education, la cual supera a aquellas con mayor número acumulado de publicaciones de artículos.

Al analizar las revistas con mayor número de citas promedio por documento publicado en las cinco décadas, se destacan: Whither Opportunity?: Rising Inequality (314); Rationality and Society (288); Impossible Bodies (280); Annual Review of Sociology (219); y, School Trouble: Identity (313).

Al analizar las publicaciones según el país de origen, Estados Unidos (28.2\%), Reino Unido (16.9\%), Alemania (13.4\%), Australia (4.5\%), China (4.2\%), Brasil (4.0\%) y Holanda (3.9\%) concentran el $75.1 \%$ del total de las publicaciones entre 1974 y 2019 . De acuerdo con la distribución a nivel internacional, se destaca el liderazgo de los países anglosajones en la producción de conocimiento en el campo de las desigualdades educativas, especialmente el aporte de los Estados Unidos (figura 1). 
Figura 1.

Distribución de las publicaciones por países entre 1974 y 2019
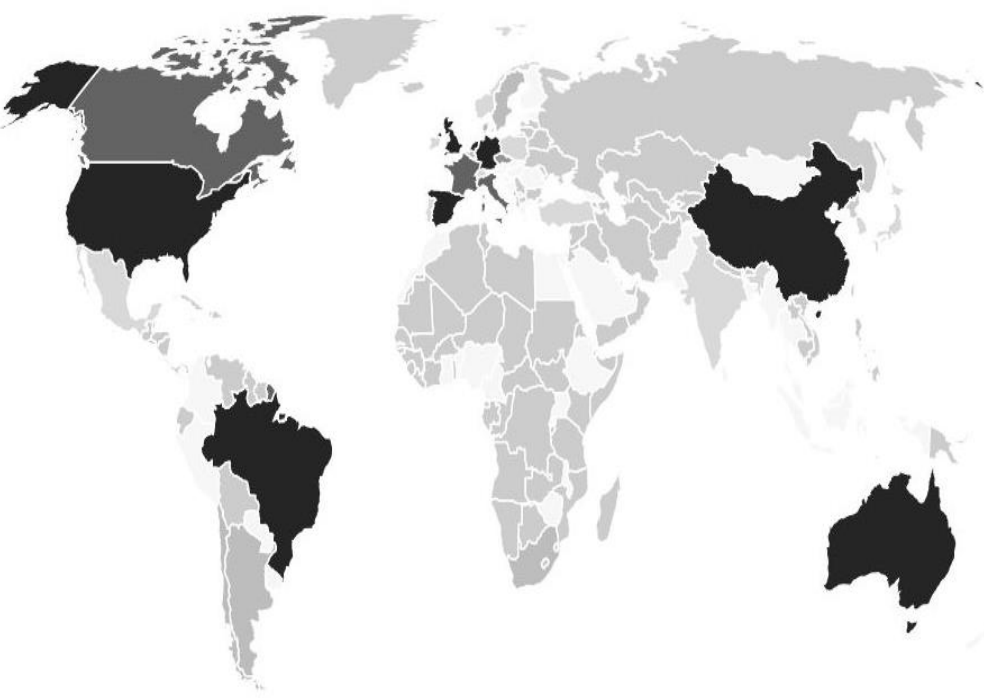

Número de documentos

Nota. Elaboración propia (2021)

Complementario a la distribución de las publicaciones por países, en el análisis de las áreas que convergen al desarrollo del campo de las desigualdades sociales destacan tres momentos en el tiempo en los que se crean puntos de inflexión donde hacen presencia otras disciplinas para analizar la desigualdad (gráfico 3).

En el gráfico 3 se describen las áreas del conocimiento asociadas a las ciencias sociales según el número de documentos y el año de publicación. El primero punto de inflexión en la producción de documentos científicos ocurre a finales de la década de los ochenta y principios de los noventa en los que el enfoque multidisciplinario contribuyó al análisis de las desigualdades educativas. Un segundo punto ocurre entre el 2003 y 2006 en el que las artes y las humanidades aportan el desarrollo del campo. Finalmente, un tercer punto de inflexión ocurrió entre 2009 y 2012, período en el que se genera una amplia participación de los campos como la economía y la psicología y se fortalecen los aportes de las artes y las humanidades. 
Gráfico 3.

Análisis de la convergencia de áreas del conocimiento al campo de la desigualdad educativa entre 1974 y 2019

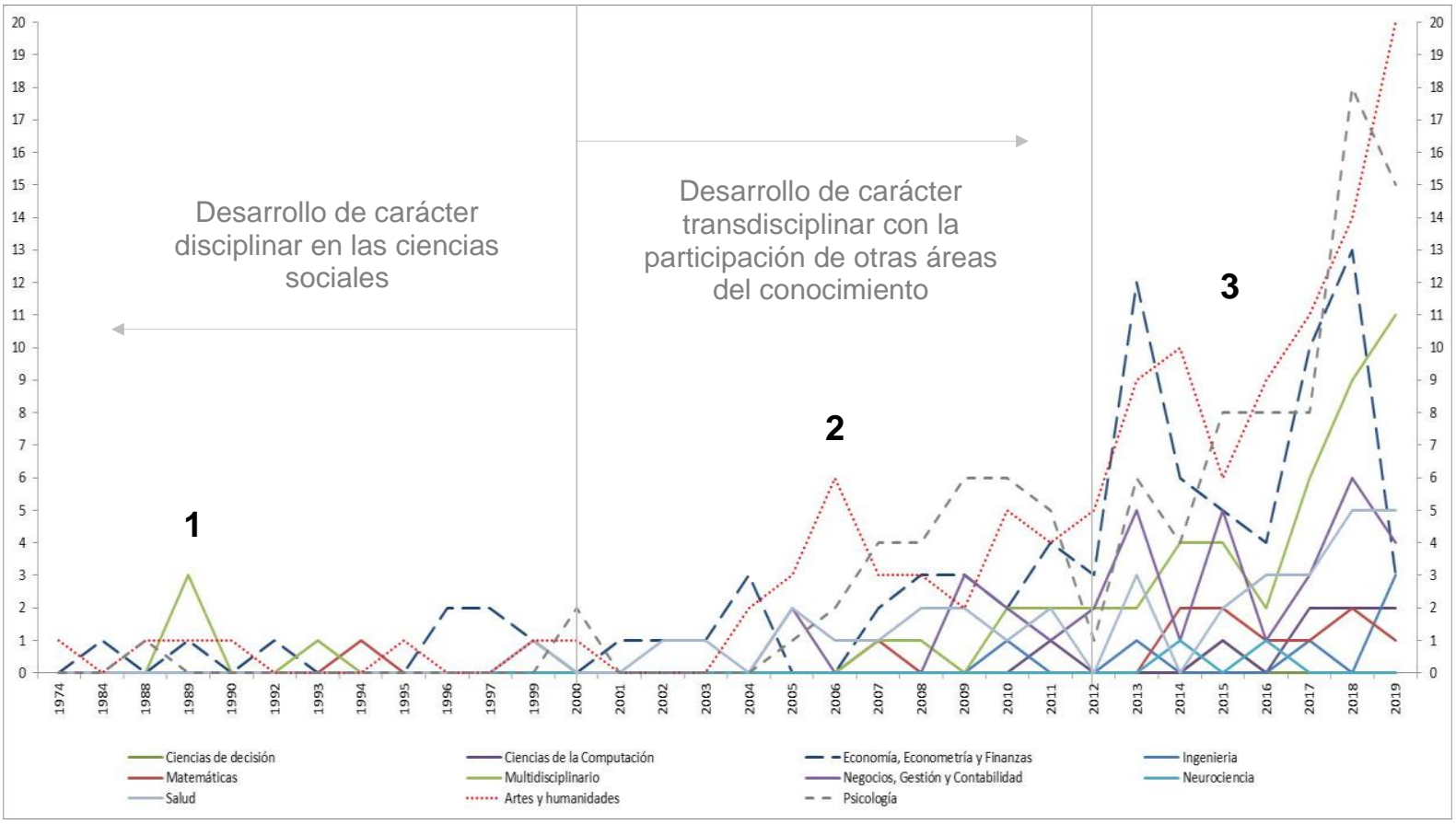

Nota. Elaboración propia (2021)

En el campo de la economía, el artículo de mayor disrupción fue Educational inequality and the expansion of UK higher education de Blanden \& Machin, el cual fue publicado en 2004 por el Scottish Journal of Political Economy y recibió 173 citas. En contraste, desde la psicología, el artículo más disruptivo fue Social origin and educational participation. Empirical analyses of educational inequality by social origin from 1950 to 1989 de Schimpl-Neimanns, publicado en 2000 en el Kolner Zeitschrift fur Soziologie und Sozialpsychologie, donde recibió 76 citas.

\section{Análisis de granularidad de los autores}

El análisis de la granularidad de los autores facilita la comprensión de la manera en que su producción de conocimiento en el campo de las desigualdades sociales se consolida según el impacto de su producción científica. Este tipo de análisis, incorporado a través de un mapa estratégico de palabras claves, permite identificar el impacto de datos agregados de una variable en el campo de conocimiento, consolidando nubes de puntos que establecen sendas de desarrollo intelectual en los temas motores, especializados, emergentes o salientes (Franceschini \& Maisano, 2011; Neumuth, Loebe \& Jannin, 2012; Li, et at, 2017; Rotolo, Rafols, Hopkins \& Leydesdorff, 2017). 
El mapa estratégico es un espacio bidimensional construido a partir de la centralidad y densidad de las palabras claves dividido en cuatro cuadrantes. La centralidad mide el grado de relación entre el descriptor, o palabra clave, y otras redes temáticas, cuantificando la fuerza de los lazos externos con otros temas. Esto permite resaltar la importancia de un descriptor en el desarrollo de todo el campo de investigación. En contraste, la densidad permite calcular la fuerza de los lazos internos del descriptor con otros descriptores dentro del campo de estudio y se interpreta como una medida del desarrollo del descriptor en el tema específico de estudio (Callon, Courtial \& Laville; 1991).

Se eligió el indicador de q2 como indicador de producción de conocimiento e impacto para el mapa estratégico, por considerarlo más completo ya que mide el impacto global de las publicaciones de un autor en un campo, diferenciándose así de otros indicadores como el índice $h$, el índice $g$, el índice gho el índice i10. Este mapa estratégico se elaboró tomando como periodo de inicio la década de los noventa debido a que en las décadas anteriores no se consolidaron autores con suficiente número de documentos que permitieran su inclusión (gráfico 4).

Al comparar la producción global de los autores, se observa que solo a partir de la década de noventa se conforma un corpus de autores que dan forma en el tiempo al campo de la desigualdad educativa. El mapa estratégico de autores en la década de los noventa describe a Jaret; Van der Berg; Rau y Demack como los autores que promovieron el mayor desarrollo. En contraste, para este período, autores como Ball y Leathwood se proyectan como autores potenciales de la década (gráfico 4a).

Para la década del 2000 al 2009, autores como Quinlivan; Kim; Walsemann; Jaffry; Fram y Barr fueron el centro del desarrollo teórico práctico y Witte, Becker; Preston; Van De Werfhorst y Kogan, se destacan como potenciales para el desarrollo del campo (gráfico 4b). Entre 2010 y 2019 Thirutnurthy; Escobar; Ferreira; Gabay-Egozi; LaFree; Almquist y Hadjar fueron el centro del desarrollo del conocimiento en el campo (gráfico 4c). 


\section{Gráfico 4.}

Diagrama estratégico de autores por 92 por décadas

a) 1990-1999

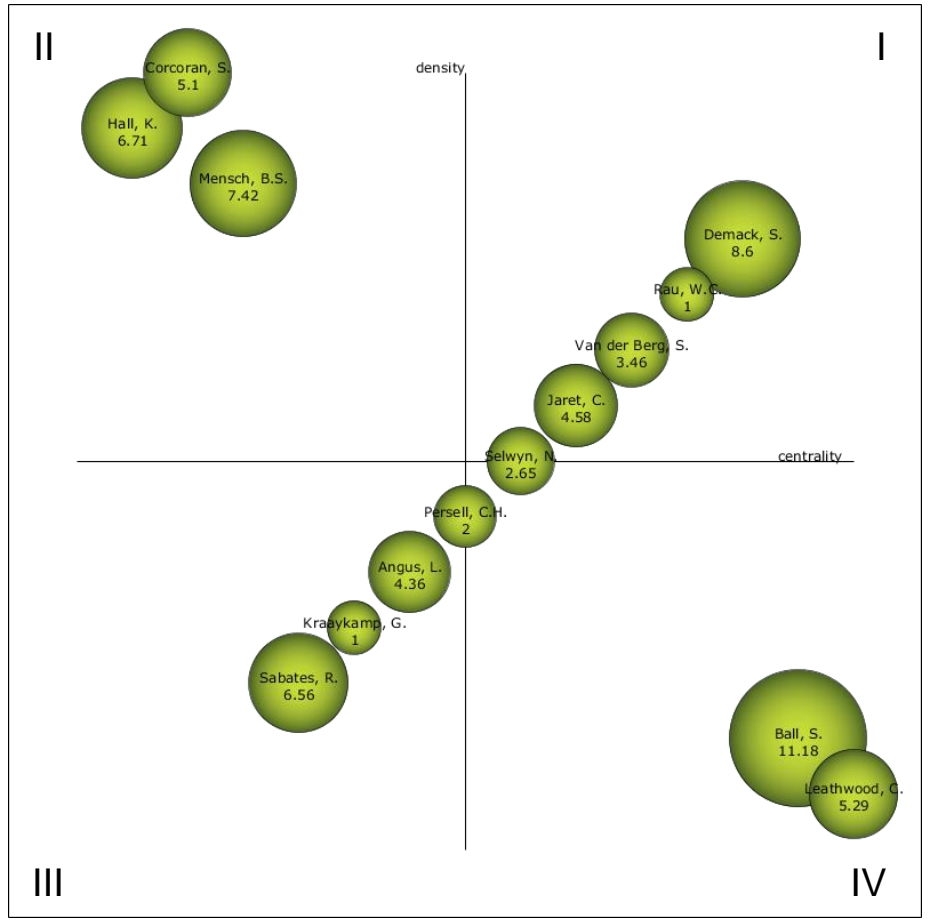

b) $2000-2009$

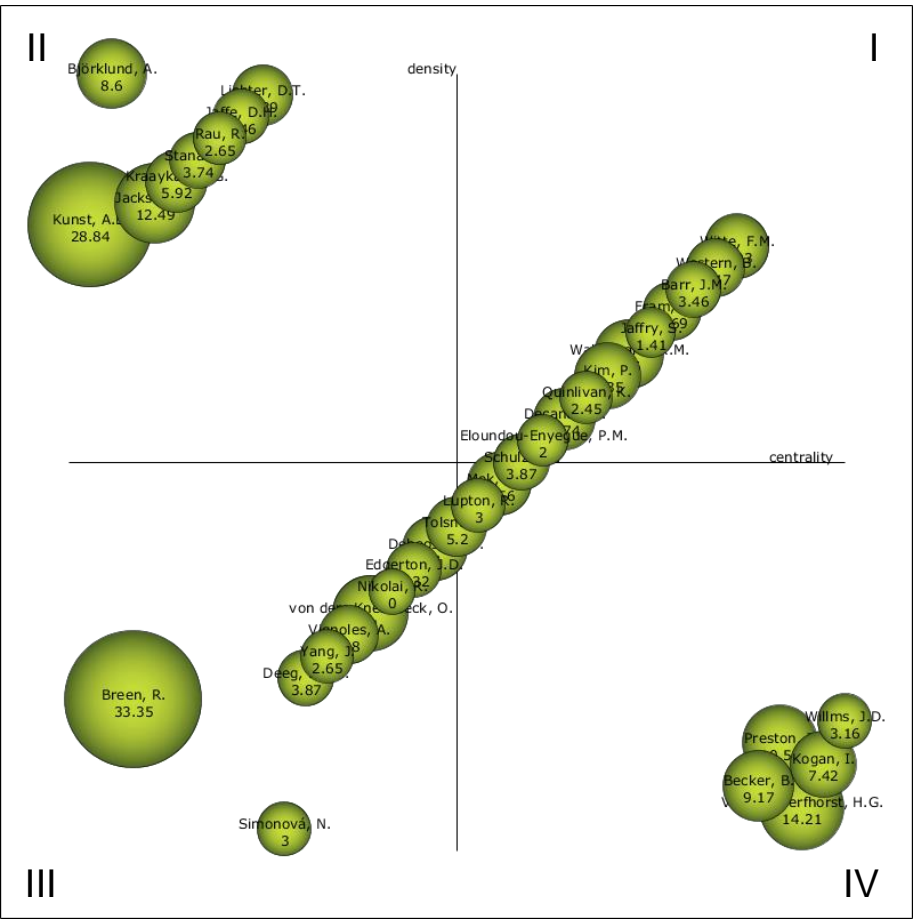

\section{c) $2010-2019$}

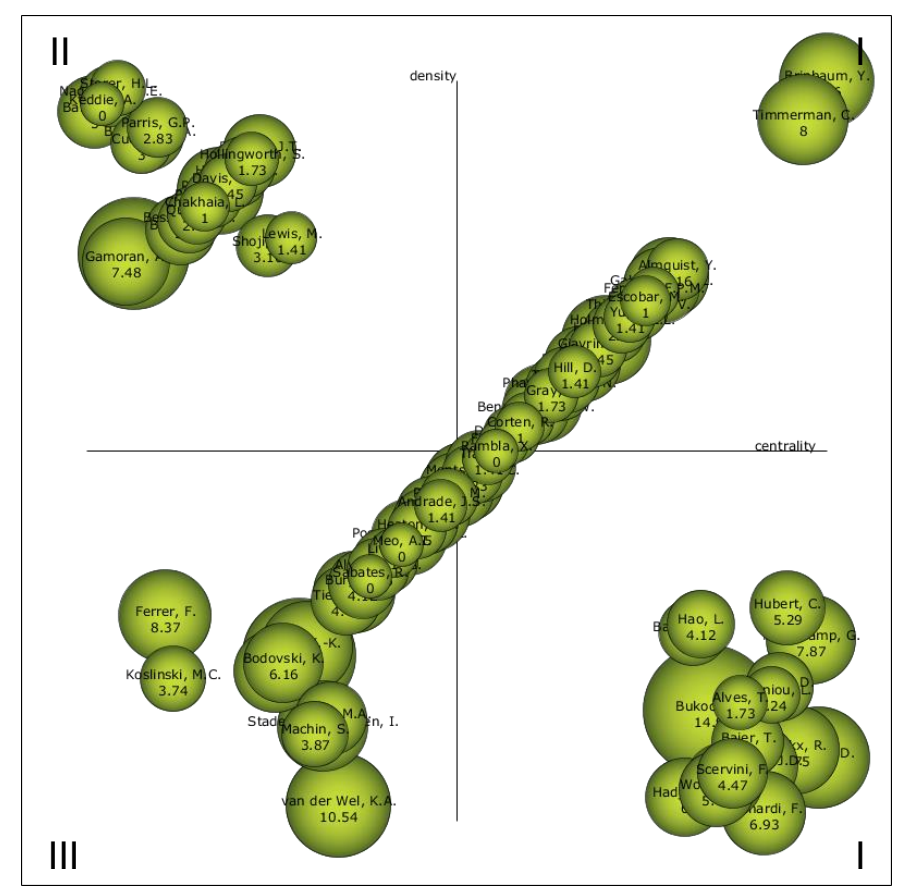

Nota. Elaboración propia (2021) 
Entre 1990 y 2019, los autores que han sido centrales para desarrollar el campo de estudio de la desigualdad educativa fueron Kilpi-Jakonen; Kunst; Jerrim; Bukodi; Crone, StrakovÃi; Reime; Jerrim; Park; Barone; Zelmanova y Kraaykamp. Estos autores tienen en común el desarrollar lazos externos con otros campos del conocimiento para aportar al estudio del análisis de las desigualdades educativas. En contraste, Njoh; Domanski; Exeter; Cochran-Smith; Storer; Kunst; Keddie; Guo; Helakorpi; Timmerman; Corcoran y Bautista, han sido centrales en el desarrollo interno del campo de estudio, es decir desarrollar aspectos centrales propios de la desigualdad.

\section{Análisis de las palabras claves}

El análisis de palabras claves inicia con la elaboración de un mapa de superposiciones que describe las medidas de estabilidad del campo de las desigualdades educativas entre 1974 y 2019. Este mapa se divide en cinco subperíodos en los que se identifica el cambio en el número de palabras calves a lo largo del tiempo analizado. Cada uno de los círculos del mapa representa un período de estudio y su número es el total de palabras claves (Figura 2).

Figura 2.

Mapa de superposiciones de palabras claves entre 1974 y 2019

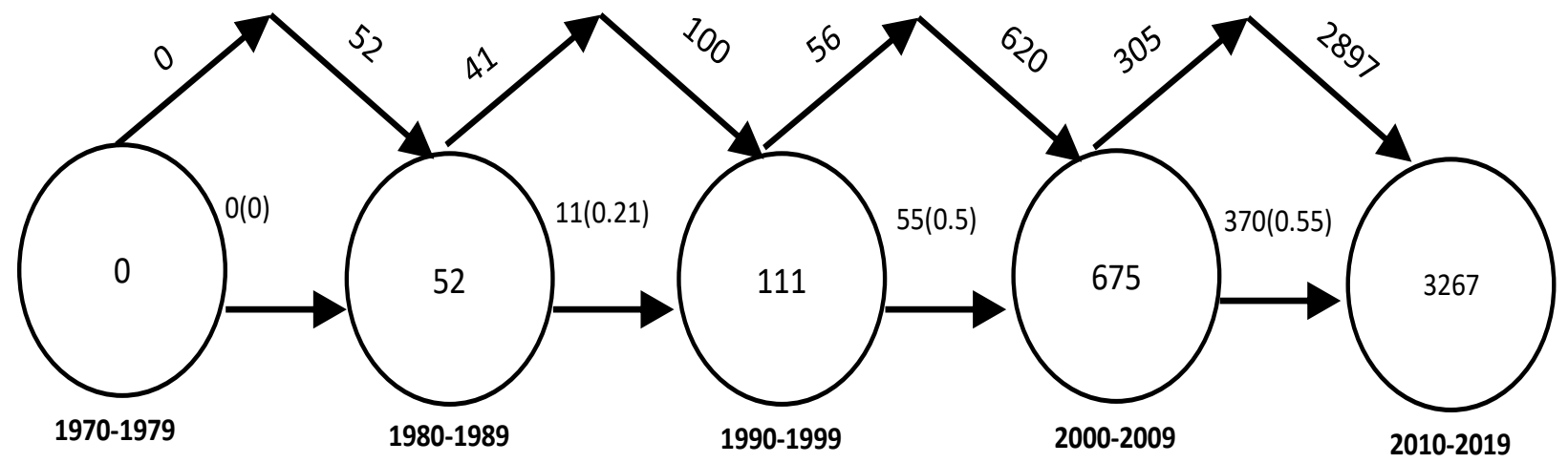

Nota. Elaboración propia (2021)

A lo largo de la ventana de observación definida, se evidencia un incremento de 0 a 3.267 palabras claves. El número de palabras claves compartido entre cada período, valor en la flecha horizontal, destaca como entre la década del setenta y el ochenta se compartieron 0 palabras claves hasta llegar a 370 entre el 2010 y el 2019.

El índice de similitud de descriptores entre períodos (valor del paréntesis), muestra un incremento de $21 \%$ en $1980-1989$ a $55 \%$ en 2019 . Este valor señala el grado en que se asemejan los descriptores temáticamente. A mayor índice, mayor similitud entre los términos. Para el campo de las desigualdades educativas, el índice señala la consolidación de palabras calves relacionadas temáticamente a lo largo del período, lo que significa que 
los términos guardan relación semántica entre sí.

Las flechas en diagonal que ingresan a cada círculo describen el número de descriptores nuevos en cada período. En este aspecto, se observa un incremento sostenido a partir de 1980 hasta el 2019.

Respecto a los descriptores que salen en cada período, flecha diagonal que sale de cada círculo, se observa una salida relativamente baja de términos en el período 1980-2019. Este comportamiento en el ingreso y egreso de términos refleja una consolidación de términos en la comunidad académica asociada al campo de estudio y a su vez, una amplia gama de relaciones con otros temas de estudio que reflejan interdisciplinariedad en el campo.

Para analizar la relación entre los descriptores, los temas y el campo de estudio de la desigualdad educativa se presenta el mapa estratégico de palabras claves (en este mapa se omite la década del setenta debido a que no contó con registros de palabras claves que permitieran su inclusión).

En el mapa estratégico (gráfico 5), se destaca como entre la década del ochenta y el noventa no se generó una mayor dispersión en las palabras claves. En la década del ochenta (gráfico 5a) se observa la existencia de dos palabras claves, desarrollo económico y desigualdad educativa. Respecto al primero, este concepto describe una alta densidad sin centralidad, lo que significa que tenía fueres vínculos respecto a los temas internos al campo, pero no establece relación con temas centrales. Por tanto, esta dinámica explica su baja contribución al desarrollo temático transdisciplinar. 
Gráfico 5.

Diagrama estratégico de palabras claves por promedio de citas por décadas

a) $1980-1989$

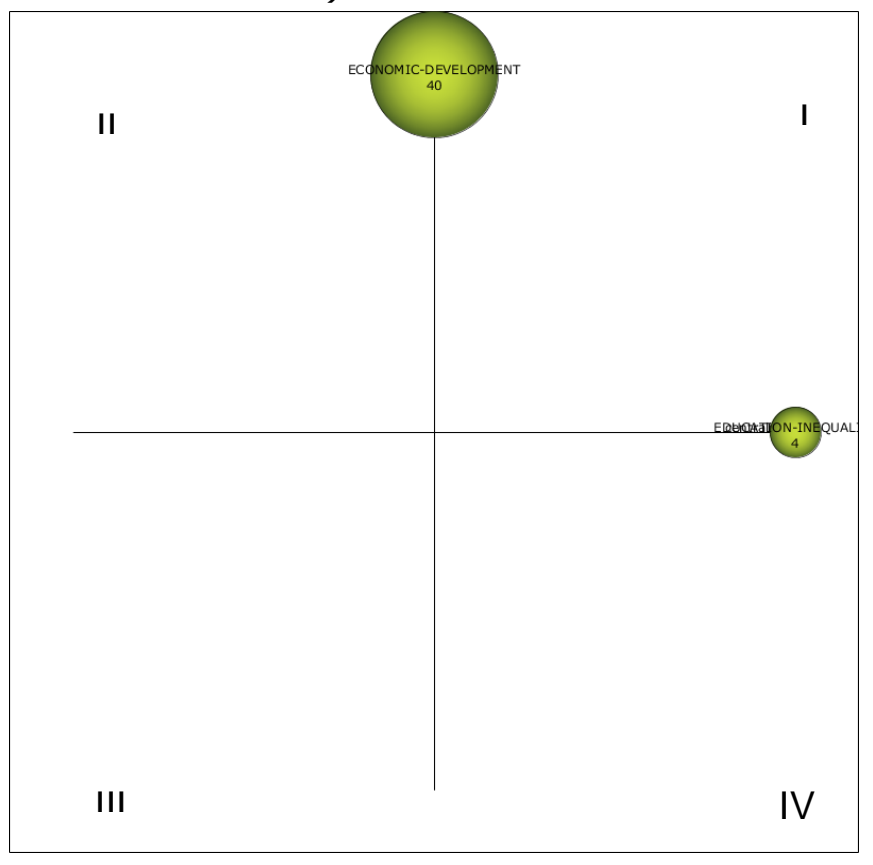

c) $2000-2009$

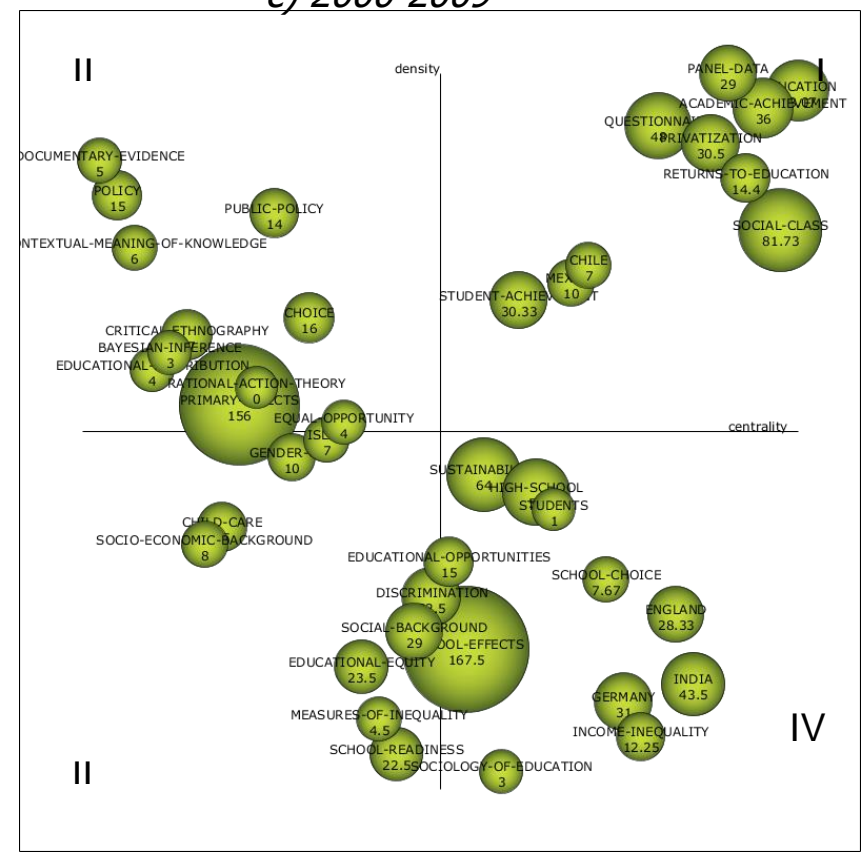

b) $1990-1999$

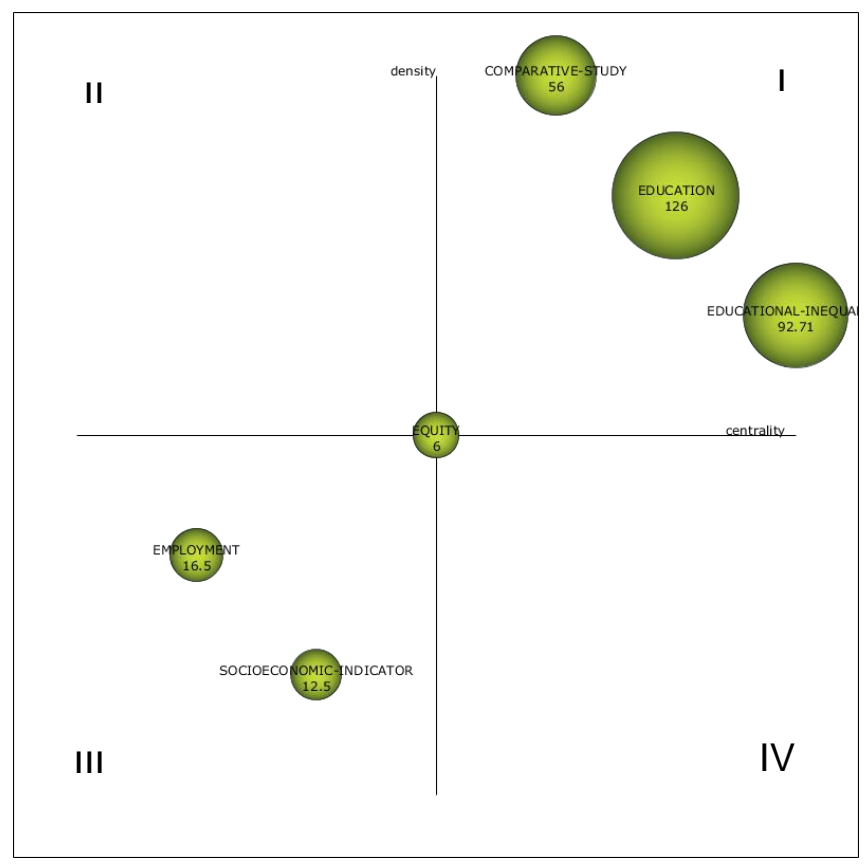

d) 2010- 2019

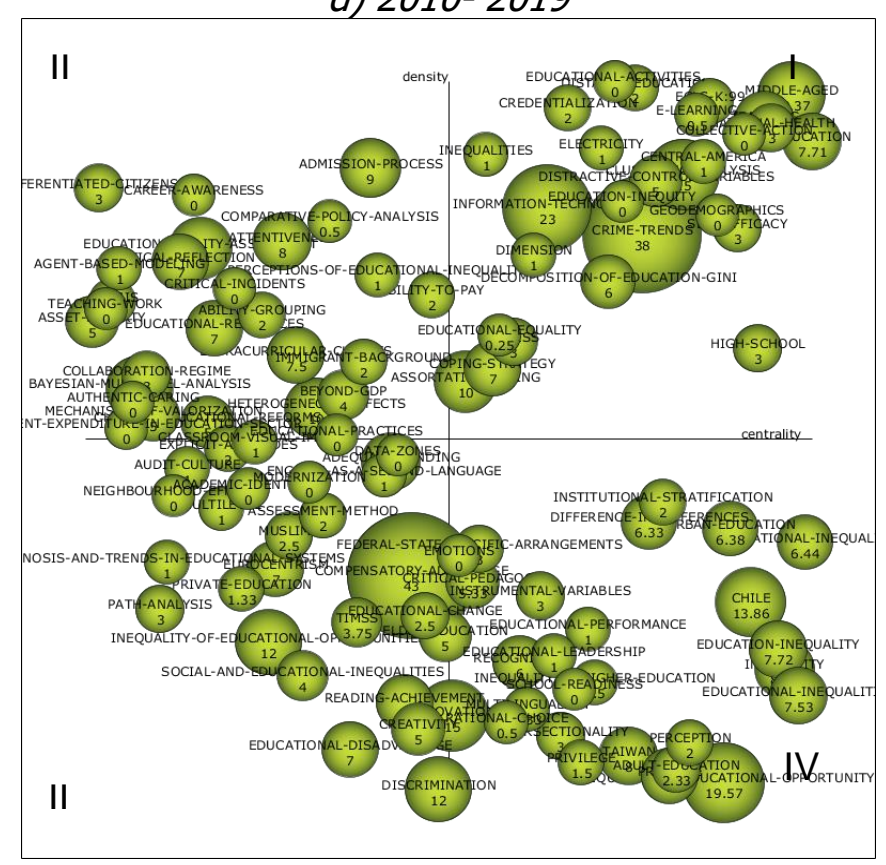

Nota. Elaboración propia (2021) 
En contraste, el tema de desigualdad educativa describe una alta centralidad, pero no densidad, lo que significa que es un tema central de estudio en el campo, pero no se relaciona con otros temas internos que fortalecen el campo. El que los cuadrantes III y IV no incluyan ninguna palabra clave significa que no existen temas marginales al campo (cuadrante III) y que no existían temas prospectivos para el desarrollo del campo en la década de los ochenta (cuadrante IV).

Por lo anterior, el análisis muestra que la década de los ochenta no aportó estructuralmente al desarrollo del campo de estudio de las desigualdades educativas, esto producto de la dispersión en los tipos de investigación o de la baja consolidación de un cuerpo de trabajo analítico desde la década anterior.

Respecto a la década del noventa (gráfico 5b), el cuadrante I destaca los estudios comparativos, la desigualdad educativa y la educación como los ejes centrales y temas motores del campo de la desigualdad educativa. Esto significa que a partir de esta década inicia el proceso de consolidación del campo de estudio con nuevos enfoques o temas de apoyo teórico práctico.

Para la década del noventa (cuadrante III), palabras claves como desempleo o indicadores económicos son marginales al campo, los cuales no describen aportes al desarrollo temático. A partir de los períodos 2000-2009 y 2010-2019 se observa la consolidación del campo temático (gráfico $5 c$ y 4 d).

Palabras claves como educación, clase social, retornos de la educación, logro académico, privatización, logro estudiantil, desigualdad de ingresos, trasfondo social o metodologías que incluyen panel de datos para el análisis, se configuraron como motores de desarrollo entre el 2000 y el 2009. En contraste, fueron temas emergentes, o prospectivos, elección escolar, sostenibilidad, oportunidades educativas, estudiantes o discriminación.

Finalmente, en el período 2010-2019, el campo de estudio describe una consolidación de un amplio grupo de palabras claves. Al respecto, en los temas motores del campo se incluyeron el nivel educativo, la edad, la salud maternal, la geo-demografía o las tendencias del crimen. Con relación a los temas prospectivos, en ellos se destacaron el estudio de las desigualdades sociales, el análisis comparativo de políticas, las clases extracurriculares, las prácticas educativas, el análisis multi nivel, las variables instrumentales o el rendimiento educativo.

Al analizar el desarrollo de las palabras claves en las cuatro décadas, se observa que el período de mayor desarrollo conceptual fue entre el 2000 y el 2019. En este período se observan nuevas metodologías, especialmente el análisis cuantitativo como el bayesiano y la relación con otras variables no económicas como las sociales, las institucionales o las demográficas. 


\section{Identificación de los ejes centrales de desarrollo de la desigualdad educativa}

El mapa estratégico de palabras claves describe la mayor producción de documentos científicos entre el período 2005 y 2019, alcanzando un 91\% del total publicado desde 1974. Con ello, este intervalo de quince años se consolida como el de mayor desarrollo científico del campo. Para identificar la evolución de este período, se analizará la evolución temática en el estudio de la desigualdad educativa (gráfico 6).

En el análisis por períodos 2005-2009, 2010-2014 y 2015-2019 se observa el desarrollo de diferentes temas. Entre el 2005 y 2009 se identifican seis temas centrales representados por círculos: escuela primaria, cuidado infantil, curso de vida, brechas de género, resultado educativo y moralidad. El tamaño de cada tema (o círculo), representa el número de documentos (centrales y/o secundarios) que acogen esta palabra clave, a mayor/menor tamaño más/menos documentos se adscriben al tema.

Entre 2010 y 2014 se identifican trece temas centrales: curso de vida, análisis multinivel, socioeconómica, escuelas privadas, adolescencia, jóvenes adultos, resultado educativo, origen social, sistema educativo, escuela primaria, mercado laboral, campos de estudio e desigualdad socioeconómica. En este período, el análisis multinivel y el curso de vida son los ejes que agrupan el mayor número de documentos.

En el 2015 y 2019 se presentan doce temas centrales: escuelas, estatus socioeconómico, análisis multinivel, sistema educativo, elecciones educativas, infantes, niñas, neoliberalismo, moralidad, brechas de género, efectos vecinales y transiciones educativas. En la evolución temática de los temas en cada intervalo analizado se identifican las conexiones establecidas entre ellos. Las líneas continuas que unen los temas indican que estos comparten el mismo nombre, están etiquetados con la misma palabra clave o la etiqueta de un tema forma parte de otro tema, es decir, que existen nexos temáticos entre ellos. A medida que la inclusión entre ellos es mayor/menor, la línea es más gruesa/delgada. 
Gráfico 6.

Evolución temática del campo de la desigualdad educativa

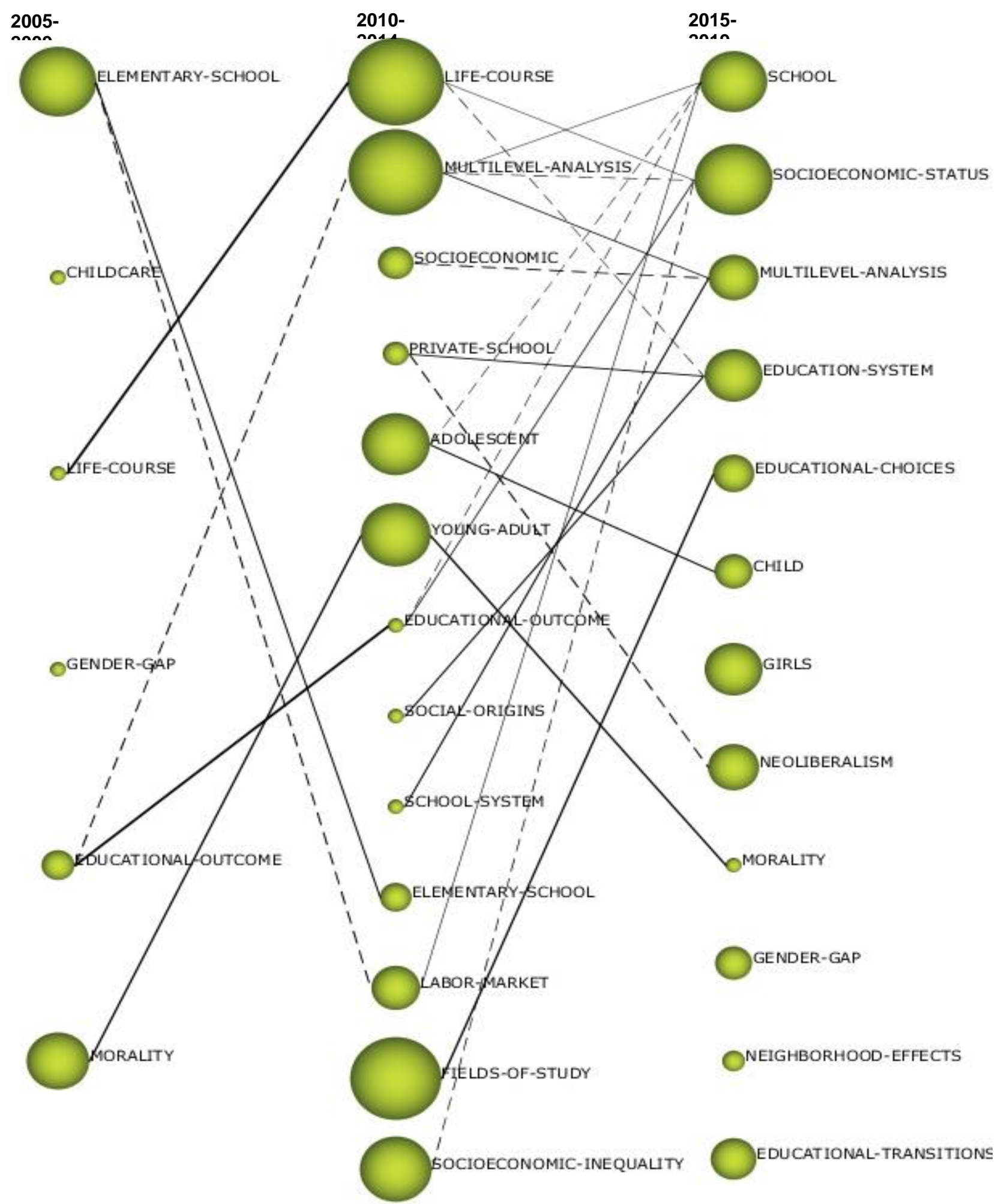

Nota. Elaboración propia (2021) 
Un ejemplo de la existencia de fuertes nexos temáticos ocurre con el tema de curso de vida o resultado educativo entre 2005-2009 y 2010-2014 donde continúan con la misma etiqueta. También con campos de estudio y elecciones educativas y jóvenes adultos, origen social y sistema educativo o moralidad entre 2010-2014 y 2015-2019.

Por otra parte, las líneas discontinuas indican que los temas comparten elementos que no están asociados a la etiqueta temática. Estos están asociados entre las categorías analíticas o metodologías, como ocurre entre curso de vida con el sistema educativo o el análisis multinivel con el estatus socioeconómico, el origen social y el análisis multinivel entre $2010-2014$ y 2015-2019.

Los temas que no muestran conexiones entre períodos, como es el caso de la brecha de género y el cuidado infantil entre 2005-2009, hacen referencia a tópicos que no se conectan con otros temas o se suspenden en el tiempo, así surjan nuevamente en otro período como es el caso de la brecha de género para el 2015-2019.

Al analizar en conjunto el período 2005-2019 se observa un desarrollo continuo en cuatro temas: i. Escuela primaria que continua en el siguiente período en la misma categoría y finaliza ahí, y se expande a mercado laboral, de donde pasa al siguiente período a la escuela; ii. Curso de vida que inicio en el primer período, siguió igual en el segundo período y se expande temáticamente al estatus socioeconómico y el sistema educativo en el último período; iii. Resultado educativo que se amplía al análisis multinivel de donde continúa en el mismo tema y se expande al estatus socioeconómico y a su vez, continua en el tema de resultado educativo y se expande a la escuela y al estatus socioeconómico en el tercer período; y, iv. Moralidad que pasa a los jóvenes adultos y de ahí, pasa a la moralidad en el tercer período.

En general, la evolución temática del campo de la desigualdad educativa se caracteriza por ser denso, especialmente entre 2010-2014 y 2015-2019, lo cual implica que los temas están interconectados, destacando diferentes áreas de formas independiente e interconectadas entre sí. Algunas de estas relaciones se reflejan en los resultados analíticos, como es el caso de Field (2013) y Aurino, Fledderjohann y Vellakkal (2019), con el estudio de las diferencias en las desigualdades educativas entre generaciones y su relación con los efectos de la inseguridad alimentaria en el aprendizaje respectivamente. Ambos autores analizan la desigualdad desde el enfoque de curso de vida, resaltando el aporte de la perspectiva longitudinal a la compresión de la desigualdad.

Kelly (2008), Verwiebe y Riederer (2013) y Schulz, Schunck, Diewald y Johnson (2017) analizaron la desigualdad educativa con el análisis multinivel de variables tales como la raza, la edad, la condición migratoria o el género, destacando con ello la integración de herramientas desde la estadística que contribuye a la identificación de variables exploratorias a la desigualdad educativa.

En contraste, Seabra (2009), D'Arcy (2014), Gaddis \& Lauen (2014), Olk (2016) y Núñez 
y Litichever (2016) analizaron la desigualdad educativa desde la perspectiva de las elecciones educativas de los estudiantes en las instituciones educativas. Para los autores, variables como la raza y la etnia, la experiencia o los antecedentes familiares contribuyen a la comprensión del origen de la desigualdad.

Tomul (2009), Matějů y Simonová (2013), Matějů \& Smith (2015), Cano, Solanas y MaríKlose (2015) y Kavita, Sharma y Parkash (2019) abordan la desigualdad educativa desde la perspectiva de las brechas de género, identificando su efecto sobre las oportunidades educativas.

\section{Discusión y conclusión}

Respecto a los interrogantes iniciales y el objetivo general propuesto, la identificación de un corpus de 1.582 documentos científicos publicados entre 1974 y 2019 permitió describir y analizar la estructura intelectual del campo de las desigualdades educativas desde una perspectiva bibliométrica, teórica y empírica.

Con los hallazgos se identificó el campo de estudio como un área de conocimiento sólida a lo largo del tiempo, principalmente con la publicación de artículos que han consolidado una postura frente al campo general de la desigual social y su enfoque se ha fortalecido con los aportes de otras disciplinas. Autores como Breen, Goldthorpe, Jonsson, Duncan, Murnane e instituciones como la Universidad de Oxford en Reino Unido y las universidades de Yale, California y Duke en Estados Unidos se consolidaron como los de mayor impacto a lo largo del período.

Revistas como Rationality and Society, Annual Review of Sociology, Whither Opportunity? Rising Inequality, Schools, and Children's Life Chances, Annual Review of Sociology y American Journal of Sociology se han consolidado como relevantes para el desarrollo del campo de la desigualdad educativa.

A lo largo del período de análisis, en la evolución del campo de estudio destacan tres etapas importantes en su configuración. Una primera etapa temática en la que el eje central era el estudio de la desigualdad educativa desde las ciencias sociales, estos estudios se caracterizaron principalmente por identificar las causas y/o consecuencias de la desigualdad en una perspectiva de la relación individuo-estructura a través de estudios de orden cualitativo y de corte trasversal en el tiempo.

Una segunda etapa se identifica con el crecimiento de estudios en campos como la economía, la psicología, la salud o las ingenierías. En esta etapa, se destaca la incorporación de metodologías cuantitativas que permiten abordar la desigualdad educativa desde distintas dimensiones y en períodos de tiempo más amplios con datos longitudinales que facilitan la identificación e interpretación de determinantes de la desigualdad. 
Finalmente, se advierte el surgimiento de una tercera etapa en la que el desarrollo de las publicaciones científicas converge a la existencia transdisciplinar de la desigualdad como un problema de investigación en el que participan múltiples áreas del conocimiento, lo que permite abordar el campo con un cuerpo teórico más amplio y multifacético que contribuye a la comprensión del fenómeno.

En general, el análisis es concluyente en resaltar los estudios de la desigualad educativa como un campo que se ha fortalecido y presenta perspectivas amplias de desarrollo a lo largo del tiempo, dando lugar así a múltiples perspectivas analíticas para abordar realidades particulares. Al respecto, se destaca la incorporación en las últimas décadas de enfoques como el de curso de vida o desempeño académico y técnicas estadísticas de mayor sofisticación de los modelos multinivel, las estadísticas bayesianas o la instrumentalización de variables asociadas a los contextos socioeconómicos que permiten indagar con mayor detalle en el contexto socioeconómico en el cual se desarrolla la desigualdad educativa, contribuyendo así a una mayor comprensión de la desigualdad social en un sentido amplio.

\section{Referencias}

Aurino, E., Fledderjohann, J. \& Vellakkal, S. (2019). Inequalities in adolescent learning: Does the timing and persistence of food insecurity at home matter? Economics of Education Review, (70), 94-108. https://doi.org/10.1016/j.econedurev.2019.03.003

Blanden, J. \& Machin, S. (2004). Educational inequality and the expansion of UK higher education. Scottish Journal of Political Economy, 51(2), 230-249. https://doi.org/10.1111/j.0036-9292.2004.00304.x

Boone, S. \& Van Houtte, M. (2013). In search of the mechanisms conducive to class differentials in educational choice: A mixed method research. Sociological Review, 61 (3), 549-572. https://doi.org/10.1111/1467-954X.12031

Boudon R. (1983). La desigualdad de oportunidades. La movilidad social en las sociedades industriales. Editorial Laia.

Bourdieu, P. \& Passeron J. C. (2009). Los herederos: los estudiantes y la cultura. Editorial Siglo XXI Editores.

Breen, R. \& Jonsson, J.O. (2005). Inequality of opportunity in comparative perspective: Recent research on educational attainment and social mobility. Annual Review of 
Sociology, (31), 223-243. https://doi.org/10.1146/annurev.soc.31.041304.122232

Callon, M., Courtial, J. P. \& Laville, F. (1991). Co-word analysis as a tool for describing the network of interactions between basic and technological research: The case of polymer chemsitry. Scientometrics, 22(1), 155-205. https://doi.org/10.1007/bf02019280

Cano, A.J., Solanas, S.E. \& Marí-Klose, M. (2015). New social risks and educational vulnerability of boys and girls in Spain [Nuevos riesgos sociales y vulnerabilidad educativa de chicos y chicas en España]. Revista de Educación, (369), 9-30. https://doi.org/10.4438/1988-592X-RE-2015-369-288

Cobo, M.J., López-Herrera, A.G., Herrera-Viedma, E. \& Herrera, F. (2011). Science mapping software tools: Review, analysis, and cooperative study among tolos. Journal of the American Society for Information Science and Technology, 62(7), 1382-1402. https://doi.org/10.1002/asi.21525

Cobo, M.J., Lõpez-Herrera, A.G., Herrera-Viedma, E. \& Herrera, F. (2012). SciMAT: A new science mapping analysis software tool. Journal of the American Society for Information Science and Technology, 63(8), 1609-1630. https://doi.org/10.1002/asi.22688

Cobo, M.J., Martínez, M.A., Gutiérrez-Salcedo, M., Fujita, H. \& Herrera-Viedma, E. (2015). 25 years at Knowledge-Based Systems: A bibliometric analysis. Knowledge-Based Systems, (80), 3-13. https://doi.org/10.1016/j.knosys.2014.12.035

Connell, R.W. (1974). The causes of educational inequality: Further observations. Journal of Sociology, 10 (3), 186-189. https://doi.org/10.1177/144078337401000306

D'Arcy, K. (2014). Home education, school, Travellers and educational inclusión. British Journal of Sociology of Education, 35(5), 818-835. https://doi.org/10.1080/01425692.2014.919840

Duncan, G.J. \& Murnane, R.J. (2011). Whither opportunity? Rising inequality, schools, and children's life chances. Whither Opportunity? Rising Inequality, Schools, and Children's Life Chances, (1), 551. 
Field, J. (2013). Learning Through the Ages? Generational Inequalities and InterGenerational Dynamics of Lifelong Learning. British Journal of Educational Studies, 61(1), 109-119. https://doi.org/10.1080/00071005.2012.756172

Franceschini, F. \& Maisano, D. (2011). Criticism on the hg-index. Scientometrics, 86(2), 339346. https://doi.org/10.1007/s11192-010-0261-1

Gaddis, S.M. \& Lauen, D.L. (2014). School accountability and the black-white test score gap. Social Science Research, 15-31. https://doi.org/10.1016/j.ssresearch.2013.10.008

Goldthorpe, J. H. (2010). De la sociología. Números, narrativas e integración de la investigación y la teoría. CIS.

Kavita, A. \& Parkash, V. (2019). Educational inequality in haryana: A study of gender differences in learning. Journal of Sociology and Social Anthropology, 10(1-3), 111120. https://doi.org/10.31901/24566764.2019/10-1-3.298

Kelley, J. (1974). The Politics of School Busing. Public Opinion Quarterly, 38(1), 23. https://doi.org/10.1086/268132

Kelly, S. (2008). Race, social class, and student engagement in middle school English classrooms. Social Science Research, 37(2), 434-448. https://doi.org/10.1016/j.ssresearch.2007.08.003

Li, L., Liu, Y., Zhu, H., Ying, S., Luo, Q., Luo, H., Kuai, X., Xia, H. \& Shen, H. (2017). A bibliometric and visual analysis of global geo-ontology research. Computers and Geosciences, (99), 1-8. https://doi.org/10.1016/j.cageo.2016.10.006

López-Herrera, A.G., Cobo, M.J., Herrera-Viedma, E., Herrera, F., Bailón-Moreno, R. \& Jiménez-Contreras, E. (2009). Visualization and evolution of the scientific structure of fuzzy sets research in Spain. Information Research, 14(4), 1-20.

Martínez, M.A., Cobo, M.J., Herrera, M. \& Herrera-Viedma, E. (2015). Analyzing the Scientific Evolution of Social Work Using Science Mapping. Research on Social Work Practice, 25(2), 257-277. https://doi.org/10.1177/1049731514522101 
Matějů, P. \& Simonová, N. (2013). Who is shortchanged in school: Boys or girls? Gender gaps in skills, grading and educational aspirations among pupils of 9th grade of elementary schools [Koho znevýhodňuje škola: chlapce, nebo dívky? Rozdíly v dovednostech, školních výsledcích a vzdilanostních aspiracích dívek a chlapců devátých tříd základních škol]. Orbis Scholae, 7(3), 107-138.

Matějů, P. \& Smith, M.L. (2015). Are boys that bad? Gender gaps in measured skills, grades and aspirations in Czech elementary schools. British Journal of Sociology of Education, 36(6), 871-895. https://doi.org/10.1080/01425692.2013.874278

Nerur, S.P., Rasheed, A.A. \& Natarajan, V. (2008). The intellectual structure of the strategic management field: An author co-citation analysis. Strategic Management Journal, 29(3), 319-336. https://doi.org/10.1002/smj.659

Neumuth, T., Loebe, F. \& Jannin, P. (2012). Similarity metrics for surgical process models. Artificial Intelligence $\quad$ in https://doi.org/10.1016/j.artmed.2011.10.001

Núñez, P. \& Litichever, L. (2016). Being young in secondary- school: Temporalities and meanings of school experiences in Argentina [Ser joven en la escuela: Temporalidades y sentidos de la experiencia escolar en la Argentina]. Psicoperspectivas, 15(2), 90-101. https://doi.org/10.5027/PSICOPERSPECTIVASVOL15-ISSUE2-FULLTEXT-854

Olk, T. (2016). Educational landscapes and the reduction of socio-spatial educational inequality in the city. Education, Space and Urban Planning: Education as a Component of the City, (7), 233-240. https://doi.org/10.1007/978-3-319-38999822

Pilkington, A., \& Meredith, J. (2009). The evolution of the intellectual structure of operations management-1980-2006: A citation/co-citation analysis. Journal of Operations Management, 27(3), 185-202. https://doi.org/10.1016/j.jom.2008.08.001

Ram, R. (1984). Population increase, economic growth, educational inequality, and income distribution. Some recent evidence. Journal of Development Economics, 14(3), 419- 


\section{8. https://doi.org/10.1016/0304-3878(84)90069-5}

Ramos-Rodríguez, A.-R., \& Ruiz-Navarro, J. (2004). Changes in the intellectual structure of strategic management research: A bibliometric study of the Strategic Management Journal, 1980-2000. Strategic Management Journal, 25(10), 981-1004. https://doi.org/10.1002/smj.397

Rotolo, D., Rafols, I., Hopkins, M.M. \& Leydesdorff, L. (2017). Strategic intelligence on emerging technologies: Scientometric overlay mapping. Journal of the Association for Information Science and Technology, 68(1), 214-233. https://doi.org/10.1002/asi.23631

Schimpl-Neimanns, B. (2000). Social origin and educational participation. Empirical analyses of educational inequality by social origin from 1950 to 1989 [Soziale herkunft und bildungsbeteiligung. Empirische analysen zu herkunftsspezifischen bildungsungleichheiten zwischen 1950 und 1989] Kolner Zeitschrift fur Soziologie und Sozialpsychologie, 52(4), 636-669.

Schulz, W., Schunck, R., Diewald, M. \& Johnson, W. (2017). Pathways of Intergenerational Transmission of Advantages during Adolescence: Social Background, Cognitive Ability, and Educational Attainment. Journal of Youth and Adolescence, 46(10), 2194-2214. https://doi.org/10.1007/s10964-017-0718-0

Seabra, T. (2009). Educational inequalities and social inequalities [Desigualdades escolares e desigualdades sociais]. Sociologia, Problemas e Praticas, (59), 75-106.

Tomul, E. (2009). Measuring regional inequality of education in Turkey: an evaluation by Gini index. Procedia - Social and Behavioral Sciences, 1(1), 949-952. https://doi.org/10.1016/j.sbspro.2009.01.168

Van De Werfhorst, H.G. \& Mijs, J.J.B. (2007). Achievement inequality and the institutional structure of educational systems: A comparative perspective. Annual Review of Sociology, (36), 407-428. https://doi.org/10.1146/annurev.soc.012809.102538

Verwiebe, R. \& Riederer, B. (2013). The reading literacy of immigrant youth in western societies: A multilevel analysis based on PISA 2000 to 2009 [Die Lesekompetenzen 
von Jugendlichen mit Migrationshintergrund in westlichen Gesellschaften: Eine Mehrebenenanalyse auf Basis der PISA-Studie von 2000 bis 2009]. Zeitschrift fur Soziologie, 42(3), 201-221.

White, H.D. \& Griffith, B.C. (1981). Author cocitation: A literature measure of intellectual structure. Journal of the American Society for Information Science, 32(3), 163-171. https://doi.org/10.1002/asi.4630320302 